\title{
UAV Flight Path Control Using Contraction-Based Backstepping Control
}

\author{
Tan Chun Kiat ${ }^{1}$, Hungsun Son ${ }^{1}$, Paw Yew Chai ${ }^{2}$ \\ ${ }^{1}$ School of Mechanical and Aerospace Engineering, Nanyang Technological University, Singapore \\ ${ }^{2}$ DSO National Laboratories, Singapore \\ Email: cktan5@ntu.edu.sg,hsson@ntu.edu.sg,pyewchai@dso.org.sg
}

Received 2013

\begin{abstract}
In this paper, a contraction-based backstepping nonlinear control technique was proposed. The proposed controller synthesis technique utilizes both the recursive nature of backstepping control and of contraction analysis. This approach results in a contracting closed-loop dynamics, with exponential stability. The use of the hierarchical contraction form in the control problem formulation also results in the exponential convergence of controlled variables and can be easily applied to non-autonomous systems. A flight path angle controller was synthesized and simulated using the proposed technique to demonstrate the exponential convergence achieved by the backstepping controller design.
\end{abstract}

Keywords: Backstepping; Contraction Theory

\section{Introduction}

In recent years, missions performed by unmanned aerial vehicle (UAV) has increasingly emphasized on high agility and maneuverability. An example is the tailsitter UAV $[1,2]$ which performs maneuver that covers a large pitch angle. These maneuvers typically require the UAVs to operate outside their linear operating regime, and results in nonlinear flight dynamics characteristics. Nonlinear control techniques, such as backstepping control, were developed to control and improve the performance of such UAVs [3-8].

The stability criterion for traditional backstepping control makes use of the Lyapunov stability theory [3-8]. However, the Lyapunov stability theory often result in complex Lyapunov control function and requires the knowledge of an equilibrium point or trajectory. This often hinders controller synthesis for trajectory tracking control as the equilibrium trajectory is often unknown and the system is often non-autonomous.

The contraction theory $[9,10]$ is a nonlinear stability theory and was applied to control formulation in several instances $[11,12]$. Stability in contraction theory is described as the diminishing of the effects from any perturbation to any trajectory in the state space. The contraction theory can be easily applied to non-autonomous systems without any added mathematical complexity and does not require the knowledge of any equilibrium point or trajectory. The contraction theory also provided a stronger form of stability as compared to Lyapunov sta- bility theory which can thus be applied to systems that require fast convergence.

The use of contraction theory as the stability criteria in backstepping control was introduced by Jouffryo [13] with the use of standard contraction form in backstepping control and later by Sharma et al. [14] and Zamani et al. [15]. Standard contraction forms refer to the arrangement/ connections of contracting subsystems to form a global contracting system. The contraction form used by [13-15] belongs to the feedback interconnection form [9].

In this paper, a hierarchical form [9] was used for the backstepping controller synthesis. This hierarchical form formulation, which was not commonly found in literatures, provided the basis for a subsystem level control. In this way, the backstepping control law can be designed for each subsystem recursively and satisfies global system contraction conditions with exponential convergence. Different from the feedback interconnection form used by [13-15], the hierarchical form also results in the exponential convergence of individual controlled variables. The proposed controller synthesis technique will be applied to a flight path angle control of a generic UAV.

This paper is organized as follows. Section 2 will present the formulation of a contraction-based backstepping control. Section 3 will describe the controller synthesis on the UAV flight path angle control. Simulation results for the closed-loop control with the contraction-based backstepping controller is presented in section 4 and conclusion of the paper will be given in section 5 . 


\section{Contraction-Based Backstepping}

This section provides the formulation of the contraction-based backstepping control.

\subsection{Contraction Theory}

The contraction theory is a stability tool for examining the stability of trajectories in the state space. According to contraction theory, a trajectory is stable if it "forgets" the effects from perturbations to that trajectory.

Consider a nonlinear system, $\dot{\mathbf{x}}=\mathbf{f}(\mathbf{x}, t)$, where $\mathbf{x} \in \mathbb{R}^{n}$ is the state of the system and $t$ is the time. By using the concept of virtual displacement on trajectories, the virtual dynamics can be written as

$$
\delta \dot{\mathbf{x}}=\frac{\partial \mathbf{f}(\mathbf{x}, t)}{\partial \mathbf{x}} \delta \mathbf{x}
$$

where $\delta \mathbf{x}$ is the virtual displacement of the trajectory.

We can further describe the time variation of $\delta \mathbf{x}^{\mathrm{T}} \delta \mathbf{x}$ as

$$
\frac{d}{d t}\left(\delta \mathbf{x}^{T} \delta \mathbf{x}\right)=\delta \mathbf{x}^{T} \frac{\partial \mathbf{f}(\mathbf{x}, t)}{\partial \mathbf{x}} \delta \mathbf{x} .
$$

Here, $\delta \mathbf{x}^{\mathrm{T}} \delta \mathbf{x}$ represents the squared distance between infinitesimally close trajectories. If the Jacobian, $\partial \mathbf{f}(\mathbf{x}, t) / \partial \mathbf{x}$, is uniformly negative definite, then the squared distance between trajectories reduces exponentially to zero. Hence, a trajectory is stable as neighboring trajectories converges into each other. The state space region where the Jacobian is uniformly negative definite is known as the contraction region.

\subsection{Hierarchical Connection Structure}

Contraction results can also be extended to different system connections. Contracting subsystems can be connected in different ways to obtain a globally contracting system. In this way, we can prove global contraction by examining the contraction behavior of smaller subsystems. The system that will be used in this paper is the hierarchical combination, which resemblances the series connection.

A system with a virtual dynamics of the form

$$
\frac{d}{d t}\left(\begin{array}{l}
\delta \mathbf{x}_{1} \\
\delta \mathbf{x}_{2}
\end{array}\right)=\left(\begin{array}{ll}
\mathbf{F}_{11} & 0 \\
\mathbf{F}_{21} & \mathbf{F}_{22}
\end{array}\right)\left(\begin{array}{l}
\delta \mathbf{x}_{1} \\
\delta \mathbf{x}_{2}
\end{array}\right)
$$

is known as the hierarchical combination. The system will be converging if the submatrices $\mathbf{F}_{11}$ and $\mathbf{F}_{22}$ are uniformly negative definite and $\mathbf{F}_{21}$ is bounded.

This is because subsystems

$$
\delta \dot{\mathbf{x}}_{1}=\mathbf{F}_{11} \delta \mathbf{x}_{1}
$$

and

$$
\delta \dot{\mathbf{x}}_{2}=\mathbf{F}_{21} \delta \mathbf{x}_{1}+\mathbf{F}_{22} \delta \mathbf{x}_{2}
$$

are converging since $\boldsymbol{x}_{1}$ is converging and the term $\mathbf{F}_{21} \delta \boldsymbol{x}_{1}$ in equation (5) is bounded and decreases exponentially to zero due to the convergence of $\boldsymbol{x}_{1}$. Therefore the trajectories in the entire state space in equation (3) are converging [9].

This implies that we can analyze or design a larger system by considering the contraction property of smaller subsystems and extending the results to the original system. We will use this principle in the control algorithm design.

\subsection{Control Algorithm}

Consider a strict feedback system in the form of

$$
\begin{aligned}
& \dot{x}_{1}=f_{1}\left(x_{1}, x_{2}\right) \\
& \dot{x}_{2}=f_{2}\left(x_{1}, x_{2}, x_{3}\right) \\
& \vdots \\
& \dot{x}_{n}=f_{n}\left(x_{1}, x_{2}, \cdots, x_{n}, u\right)
\end{aligned}
$$

where $x_{1}, x_{2}, \ldots, x_{n}$ are the states of the system, for the recursive backstepping control formulation.

A coordinate transformation will be carried out to transform the system into the hierarchical contraction form.

Define the transformation

$$
z_{1}=x_{1}-x_{1}^{(r)}
$$

where $x_{1}^{(r)}$ is the reference trajectory for $x_{1}$. The $z_{1}$-dynamics becomes

$$
\dot{z}_{1}=f_{1}\left(x_{1}, x_{2}\right)-\dot{x}_{1}^{(r)}
$$

where $x_{2}=g_{1}\left(x_{1}\right)$ is defined as the virtual control such that the virtual dynamics is in the form of equation (9) with $x_{1}^{(r)}$ as a particular solution.

$$
\delta \dot{z}_{1}=h_{11}\left(z_{1}\right) \delta z_{1}+h_{12}\left(z_{1}, z_{2}\right) \delta z_{2}
$$

where $z_{2}=x_{2}-g_{1}\left(x_{1}\right)$ with $h_{11}$ uniformly negative definite and $h_{12}$ bounded.

It is clear that if $\delta z_{2}$ reduces to zero, then $x_{1} \rightarrow x_{1}^{(r)}$. A similar transformation procedure is applied to the $z_{2-}$ dynamics.

$$
\dot{z}_{2}=f_{2}\left(x_{1}, x_{2}, x_{3}\right)-\frac{d}{d t} g\left(x_{1}\right)
$$

where $x_{3}=g_{2}\left(x_{1}, x_{2}\right)$ is defined as the virtual control such that the virtual dynamics is in the form of equation (11) with $x_{2}=g_{1}\left(x_{1}\right)$ as a particular solution.

$$
\delta \dot{z}_{2}=h_{22}\left(z_{2}\right) \delta z_{2}+h_{23}\left(z_{2}, z_{3}\right) \delta z_{3}
$$

where $z_{3}=x_{3}-g_{2}\left(x_{1}, x_{2}\right)$ with $h_{22}$ is uniformly negative definite and $h_{23}$ bounded.

The recursive transformation procedure is performed until the control input is obtained. The virtual dynamics for the global feedback in equation (6) can be expressed in the following form. 


$$
\frac{d}{d t}\left(\begin{array}{c}
\delta z_{1} \\
\delta z_{2} \\
\vdots \\
\delta z_{n}
\end{array}\right)=\left(\begin{array}{ccccc}
h_{11} & h_{12} & 0 & \cdots & 0 \\
0 & h_{22} & h_{23} & \cdots & 0 \\
0 & 0 & h_{33} & \cdots & 0 \\
\vdots & \vdots & \vdots & \ddots & \vdots \\
0 & 0 & 0 & \cdots & h_{n n}
\end{array}\right)\left(\begin{array}{c}
\delta z_{1} \\
\delta z_{2} \\
\vdots \\
\delta z_{n}
\end{array}\right)
$$

This is in the hierarchical contraction form that provides the global feedback system with exponential stability.

In addition, the hierarchical contraction form provides exponential convergence for each individual state, $z_{i}$ where $i=1,2, \ldots, n$. This is because the virtual dynamics for each state is

$$
\delta \dot{z}_{i}=h_{i i} \delta z_{i}+h_{i, i+1} \delta z_{i+1}
$$

where the second term can be regarded as a bounded disturbance term that diminishes to zero. In particular, the controlled variable, $z_{1}$, converges to the reference value exponentially which can be used for fast convergence control of a particular variable.

\section{UAV Flight Path Control}

\subsection{Dynamical Model}

This section provides a description of the contraction-based backstepping controller synthesis with a simplified UAV model for flight path angle control.

Figure 1 shows the forces acting on the longitudinal axis of the UAV, the dynamical model is given by equation (14) [16].

$$
\begin{aligned}
\dot{\gamma} & =\frac{1}{m V}(L+T \sin \alpha)-\frac{g}{V} \cos \gamma \\
\dot{\theta} & =q \\
\dot{q} & =M=u
\end{aligned}
$$

where $\gamma$ is the flight path angle, $\theta$ is the pitch angle, $q$ is the pitch rate, $m$ is the aircraft mass, $V$ is the air speed, $T$ is the thrust, $\alpha$ is the angle of attack, $g$ is the gravitational acceleration, $L$ is the aerodynamic lift and $M$ is the aerodynamic pitch moment which is defined as the control input $u$.

The lift force, $L$, is expressed in the following form.

$$
L=\frac{1}{2} \rho V^{2} S C_{L}(\alpha)
$$

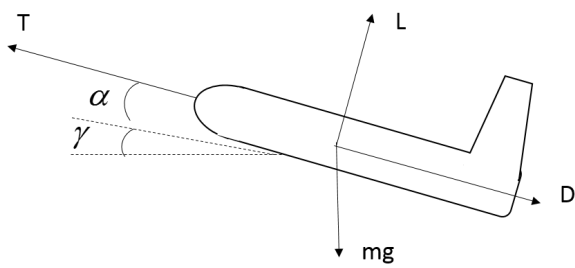

Figure 1. UAV dynamical model. where $\rho$ is the air density, $S$ is the wing area and $C_{L}$ is the lift coefficient and is a function of $\alpha$.

The following assumptions were made to simplify the model and the control formulation.

- The control surfaces only produce aerodynamic moments. The aerodynamic forces produced were assumed to be small and neglected.

- The speed of the aircraft was maintained at a constant value independently.

- The control actuator dynamics were sufficiently fast, thus neglected.

Therefore, equation (14) was reduced to the form shown in equation (16).

$$
\begin{aligned}
& \dot{\gamma}=f(\alpha, t)-\frac{g}{V} \cos \gamma \\
& \dot{\theta}=q \\
& \dot{q}=u
\end{aligned}
$$

where $f(\alpha, t)=\frac{1}{m V}(L(\alpha)+T(t) \sin \alpha)$.

Equation (16) is a simplified dynamical model for the purpose of key concept demonstration and forms the basis for the control formulation which will be presented in the next section.

\subsection{Flight Path Angle Tracking}

The above control algorithm was applied to a flight path tracking problem of an UAV.

Figure 2 shows a block diagram of the closed-loop system, highlighting the subsystem nature of the algorithm.

Step 1:

Define the transformation

$$
z_{1}=\gamma-\gamma^{*}
$$

where $\gamma^{*}$ is a reference flight path angle defined in equation (28). The $z_{1}$-dynamics becomes

$$
\dot{z}_{1}=f(\alpha, t)-\frac{g}{V} \cos \left(z_{1}+\gamma^{*}\right)-\dot{\gamma}^{*}
$$

To define a suitable virtual control, we assume that $z_{2}=\alpha-\left(-K z_{1}\right)$, where $K>0$, and consider the virtual dynamics of equation (18).

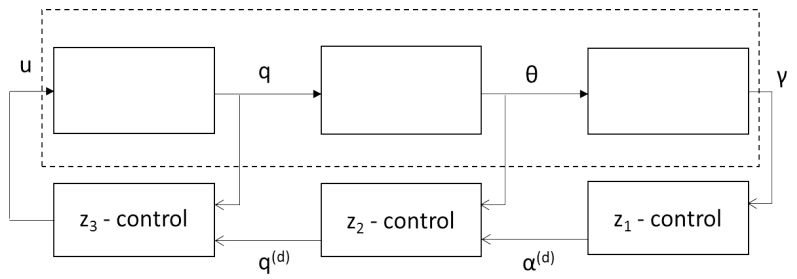

Figure 2. Block diagram of the closed-loop system for the flight path angle control problem. 


$$
\begin{aligned}
\delta \dot{z}_{1}= & {\left[-K f^{\prime}\left(z_{2}-K z_{1}, t\right)+\frac{g}{V} \sin \left(z_{1}+\gamma^{*}\right)\right] \delta z_{1} } \\
& +f^{\prime}\left(z_{2}-K z_{1}, t\right) \delta z_{2}
\end{aligned}
$$

For the subsystem in equation (19) to be in the contraction region, the operation region is limited so that $K=\frac{1}{f_{\min }^{\prime}}\left(\frac{g}{V}+K_{1}\right)$ where $K_{1}>0$, then the subsystem given by equation (19) will be in the contraction region.

Step 2:

Hence the $z_{2}$-dynamics becomes

$$
\begin{aligned}
\dot{z}_{2} & =\dot{\alpha}+K \dot{z}_{1} \\
& =q-\dot{\gamma}+K \dot{z}_{1} .
\end{aligned}
$$

If we define $z_{3}=q-q^{(d)}$ where

$$
q^{(d)}=-K_{2} \delta z_{2}+\dot{\gamma}-K \dot{z}_{1}
$$

and $K_{2}>0$, then the virtual dynamics will become

$$
\delta \dot{z}_{2}=-K_{2} \delta z_{2}+\delta z_{3}
$$

which implies a contraction region.

Step 3:

Now, consider $z_{3}$-dynamics

$$
\dot{z}_{3}=u-\dot{q}^{(d)}
$$

If we let the control input $u$ be

$$
u=\dot{q}^{(d)}-K_{3} z_{3}
$$

and $K_{3}>0$, then the virtual dynamics will become

$$
\delta \dot{z}_{3}=-K_{3} \delta z_{3}
$$

which implies a contraction region. be

Hence the virtual dynamics for the entire system will

$$
\frac{d}{d t}\left(\begin{array}{l}
\delta z_{1} \\
\delta z_{2} \\
\delta z_{3}
\end{array}\right)=\left(\begin{array}{ccc}
h_{11} & h_{12} & 0 \\
0 & -K_{2} & 1 \\
0 & 0 & -K_{3}
\end{array}\right)\left(\begin{array}{l}
\delta z_{1} \\
\delta z_{2} \\
\delta z_{3}
\end{array}\right)
$$

where $\quad h_{11}=-K f^{\prime}\left(z_{2}-K z_{1}, t\right)+\frac{g}{V} \sin \left(z_{1}+\gamma^{*}\right)$

and $h_{12}=f^{\prime}\left(z_{2}-K z_{1}, t\right)$, is in the hierarchical contraction form.

Step 4:

For the tracked angle, $\gamma^{(r)}$, to be a particular solution so that all trajectories contract onto it, the reference angle, $\gamma^{*}$, is defined as follows. Consider the resultant dynamics,

$$
\begin{aligned}
& \dot{z}_{1}=f(\alpha, t)-\frac{g}{V} \cos \left(z_{1}+\gamma^{*}\right)-\dot{\gamma}^{*} \\
& \dot{z}_{2}=-K_{2} z_{2}+z_{3} \\
& \dot{z}_{3}=-K_{3} z_{3}
\end{aligned}
$$

It can be seen that $z_{2}=z_{3}=0$ is a particular solution.
Putting the actual tracked flight path angle, $\gamma^{(r)}$, as a particular solution into the $z_{1}$-dynamics,

$$
\begin{aligned}
\dot{\gamma}^{(r)}-\dot{\gamma}^{*} & =f\left(-K\left(\gamma^{(r)}-\gamma^{*}\right)\right)-\frac{g}{V} \cos \gamma^{(r)}-\dot{\gamma}^{*} \\
f\left(\alpha^{(r)}\right) & =f\left(-K\left(\gamma^{(r)}-\gamma^{*}\right)\right) \\
\gamma^{*} & =\frac{1}{K} \alpha^{(r)}+\gamma^{(r)}
\end{aligned}
$$

where we had used the fact that

$$
\dot{\gamma}^{(r)}=f\left(\alpha^{(r)}\right)-\frac{g}{V} \cos \gamma^{(r)}, \quad z_{2}=0
$$

and the symbol $t$ is omitted in equation (28) for neatness. Note that the difference between $\gamma^{*}$ and $\gamma^{(r)}$ is due to the nonzero angle of attack, $\alpha$, at equilibrium so a nonzero $\gamma^{*}$ is required to account for this difference.

Hence the above formulated control law and reference flight path angle, $\gamma^{*}$, tracks the flight path angle, $\gamma^{(r)}$.

\section{Simulation Results}

The synthesized control law was implemented and tested in Matlab for flight path angle stabilization and tracking problem.

To study the convergence of the implemented controller, an initial perturbation (away from equilibrium) was applied to the system and the system closed-loop response was examined.

\subsection{Flight Path Angle Regulation}

The flight path angle regulation problem is the stabilization of the flight path angle to a reference value. It demonstrated the ability for the controller to reject perturbation exponentially fast. Figure $\mathbf{2}$ to $\mathbf{5}$ are obtained from the flight path stabilization simulation.

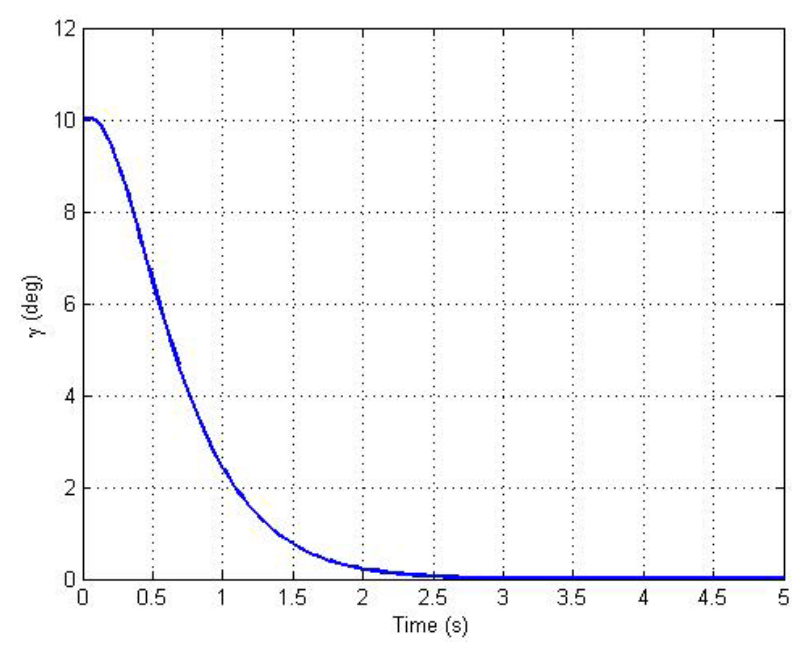

Figure 3. Flight path angle response for the stabilization problem. 


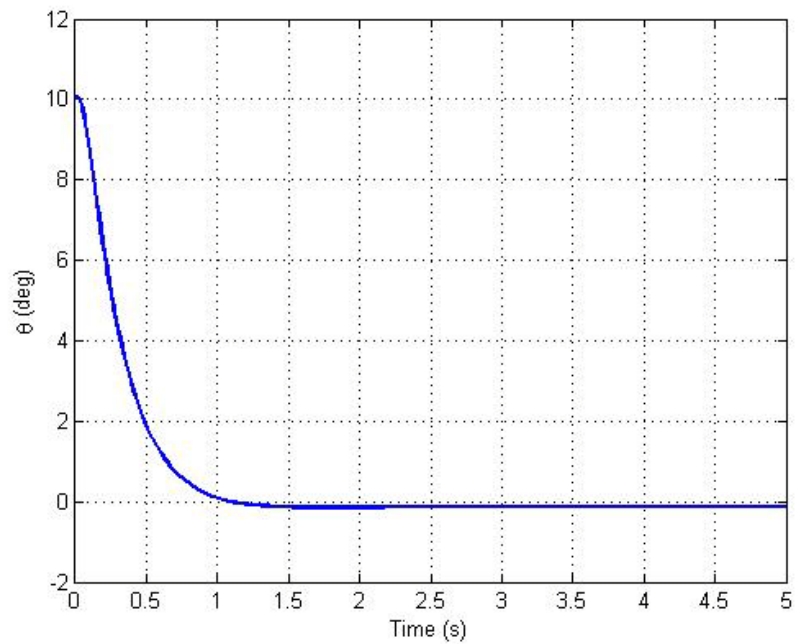

Figure 4. Pitch angle response for stabilization the problem.

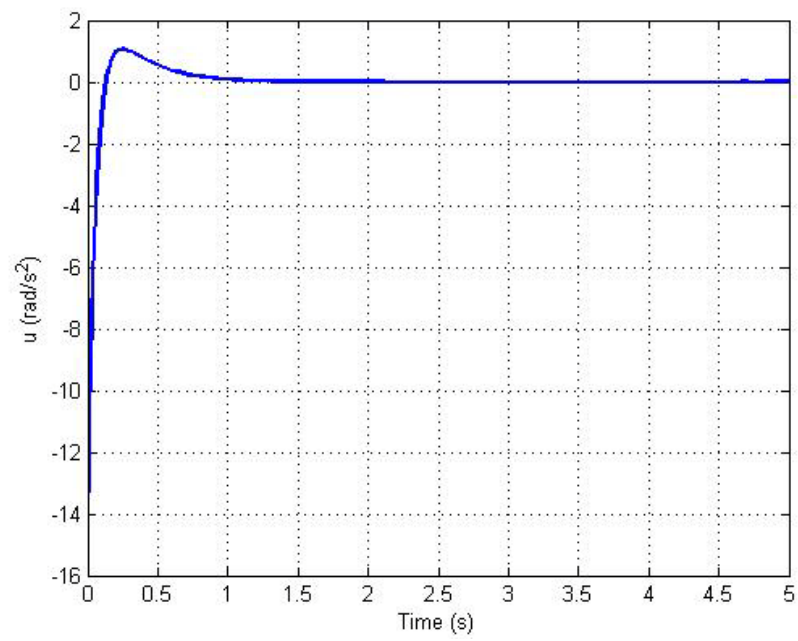

Figure 5. Control input for the stabilization problem.

\subsection{Flight Path Tracking}

In the flight path tracking problem, the ability for the controller to track time-varying reference flight path angle was demonstrated. A sinusoidal reference was chosen as an example of time-varying signal. Figure 6 to 9 shows the results for the flight path angle tracking simulation.

In both examples, it was shown that exponential stability was achieved by the control algorithm. This is due to the presence of a contraction region.

Furthermore, the controlled variable was made to be exponentially stable subjected to bounded disturbances due to the errors in virtual controls.

Although simplified, this example demonstrated the potential of the contraction-based backstepping technique as an alternative to the Lyapunov backstepping technique in the formulation of a control algorithm that achieves exponential stability in a system.

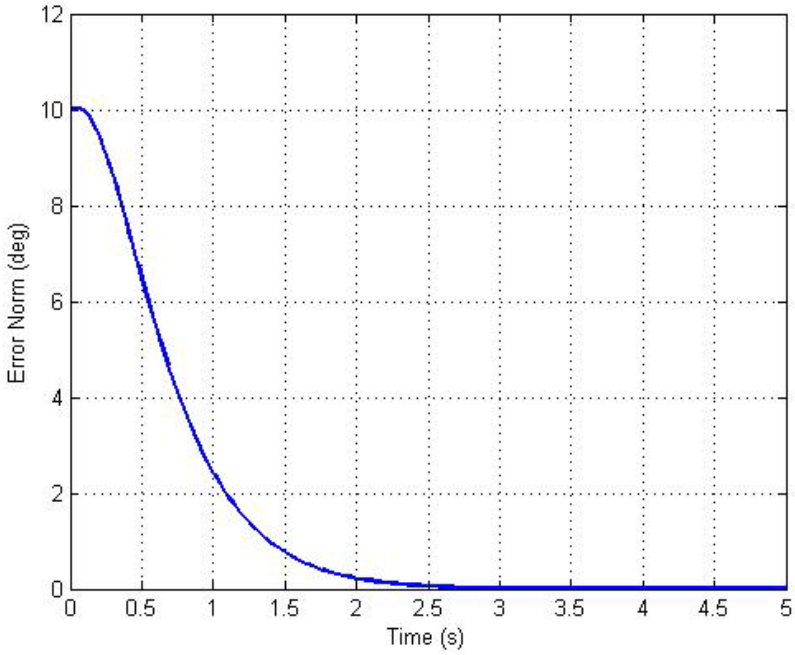

Figure 6. Flight path angle error for the stabilization problem.

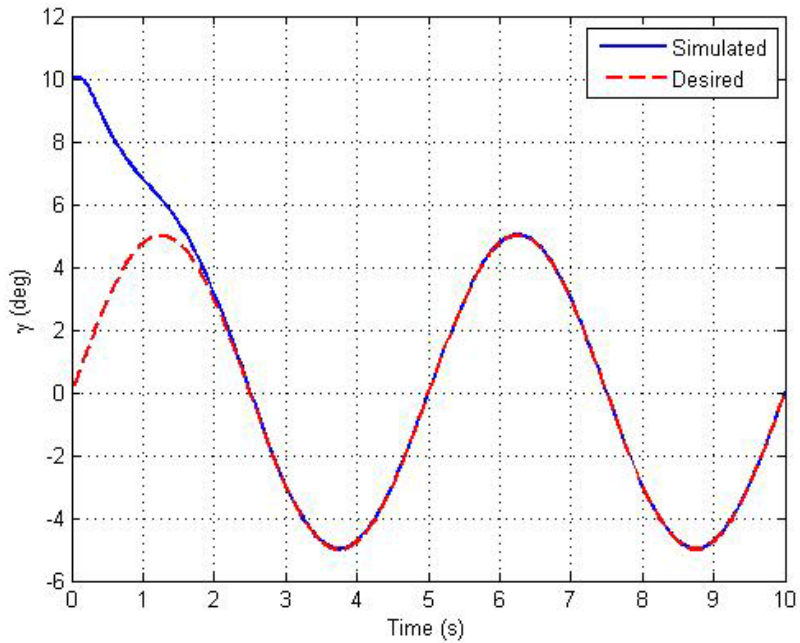

Figure 7. Flight path angle for the tracking problem.

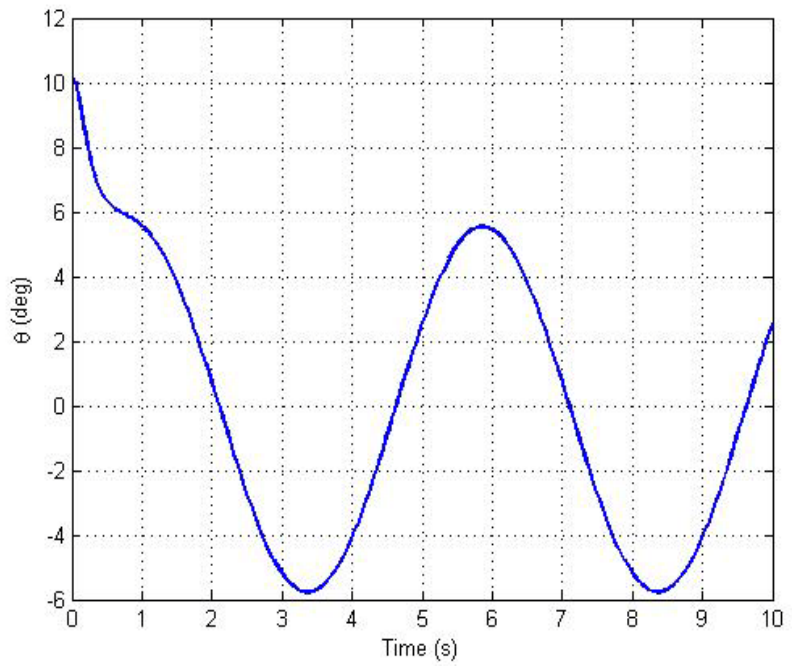

Figure 8. Pitch angle for the tracking problem. 


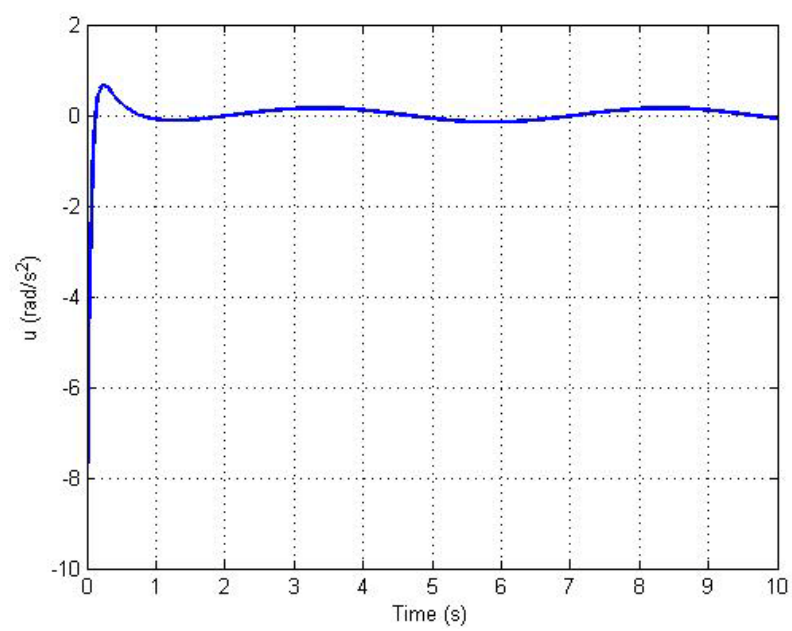

Figure 9. Control input for the tracking problem.

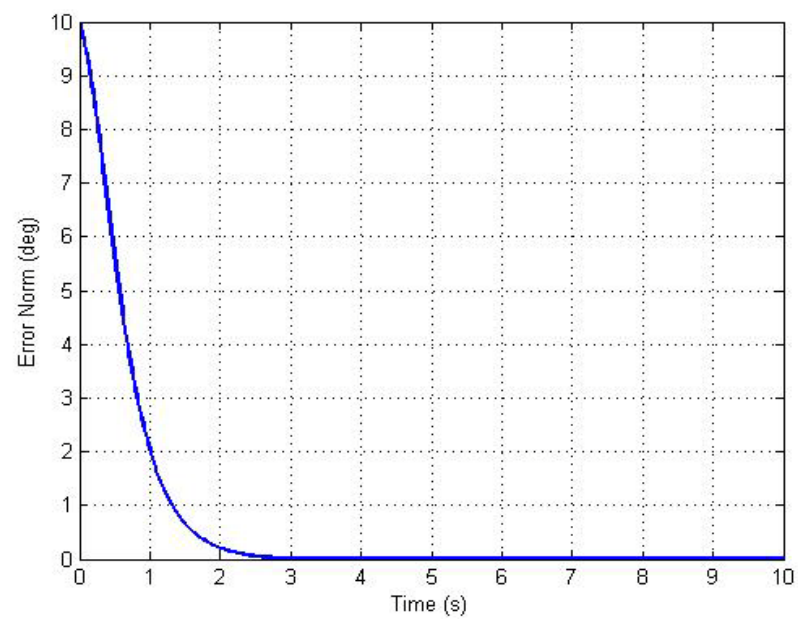

Figure 10. Flight path angle error for the tracking problem.

\section{Conclusions}

In this paper, a contraction-based backstepping technique using the hierarchical contraction structure was demonstrated. The unique hierarchical contraction structure with backstepping control formulation provides recursive control law that was formulated systematically. Exponential stability of the closed-loop system and individual controlled variable was achieved. The control algorithm was demonstrated on a flight path angle stabilization and tracking problem.

\section{Acknowledgements}

The authors would like to thank Nanyang Technological University and DSO National Laboratories for their support on this project.

\section{REFERENCES}

[1] R. H. Stone, "The T-wing Tail-Sitter Unmanned Air Ve- hicle: From Design Concept to Research Flight Vehicle," Proceedings of the Institution of Mechanical Engineers, Part G: Journal of Aerospace Engineering, Vol. 218, No. 6, 2004, pp. 417-433. doi:10.1243/0954410042794920

[2] J. Carl G. Schaefer and L. J. Baskett, "GoldenEye: The Clandestine UAV," in 2nd AIAA "Unmanned Unlimited" Systems, Technologies, and Operations, 2003, pp. 1-11.

[3] J. Escareno, R. H. Stone, A. Sanchez and R. Lozano, "Modeling and Control Strategy for the Transition of a Convertible Tail-sitter UAV," in European Control Conference, 2007.

[4] O. Harkegard, "Flight Control Design Using Backstepping," Linkoping University, 2003.

[5] N. B. Knoebel and T. W. McLain, "Adaptive Quaternion Control of A Miniature Tailsitter UAV," in 2008 American Control Conference, 2008, pp. 2340-2345. doi:10.1109/ACC.2008.4586841

[6] A. A. Mian, M. I. Ahmad and D. Wang, "Backstepping based Nonlinear Flight Control Strategy for 6 DOF Aerial Robot," in 2008 International Conference on Smart Manufacturing Application, 2008, pp. 146-151. doi:10.1109/ICSMA.2008.4505630

[7] F. M. Subolic, "Agile Flight Control Techniques for a Fixed-Wing Aircraft," Massachusetts Institute of Technology, 2009.

[8] J. H. Yang and W. C. Hsu, "Adaptive Backstepping Control for Electrically Driven Unmanned Helicopter," Control Engineering Practice, Vol. 17, No. 8, 2009, pp. 903-913. doi:10.1016/j.conengprac.2009.02.012

[9] W. S. Lohmiller, "Contraction Analysis of Nonlinear Systems," Massachusetts Institute of Technology, 1999.

[10] W. Lohmiller and J. J. E. Slotine, "On Contraction Analysis for Non-linear Systems," Automatica, Vol. 34, No. 6, 1998, pp. 683-696. doi:10.1016/S0005-1098(98)00019-3

[11] W. Lohmiller and J. J. E. Slotine, "Control System Design for Mechanical Systems Using Contraction Theory," IEEE Transactions on Automatic Control, Vol. 45, No. 5, 2000, pp. 984-989. doi:10.1109/9.855568

[12] J. Jouffroy and J. J. E. Slotine, "Methodological Remarks on Contraction Theory," 2004 43rd IEEE Conference on Decision and Control (CDC) (IEEE Cat. No.04CH37601), Vol. 3, 2004, pp. 2537-2543.

[13] J. Jouffryo, "Integrator Backstepping Using Contraction Theory: A Brief Methodological Note,” 2002, p. 238.

[14] B. B. Sharma and I. N. Kar, "Contraction Theory-based Recursive Design of Stabilising Controller for A Class of Non-linear Systems," IET Control Theory \& Applications, Vol. 4, No. 6, 2010, p. 1005. doi:10.1049/iet-cta.2009.0060

[15] M. Zamani and P. Tabuada, "Towards Backstepping Design for Incremental Stability," 49th IEEE Conference on Decision and Control (CDC), 2010, pp. 2426-2431. doi:10.1109/CDC.2010.5717210

[16] B. L. Stevens and F. L. Lewis, "Aircraft Control and Simulation," John Wiley and Sons, 2003, p. 664. 